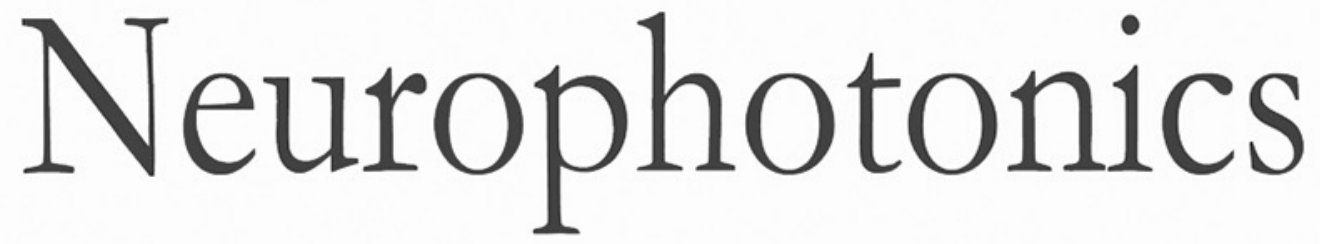

\title{
Three-dimensional visualization of intramuscular innervation in intact adult skeletal muscle by a modified iDISCO method
}

\author{
Yusha Li \\ Jianyi Xu \\ Jingtan Zhu \\ Tingting $\mathrm{Yu}$ \\ Dan Zhu
}




\title{
Three-dimensional visualization of intramuscular innervation in intact adult skeletal muscle by a modified iDISCO method
}

\author{
Yusha Li, ${ }^{\text {a,b }}$ Jianyi Xu, ${ }^{\text {a,b }}$ Jingtan Zhu,,${ }^{\text {a,b }}$ Tingting Yu, ${ }^{\text {a,b,* }}$ and Dan Zhu ${ }^{a, b}$ \\ ${ }^{a}$ Huazhong University of Science and Technology, Britton Chance Center for Biomedical \\ Photonics, Wuhan National Laboratory for Optoelectronics, Wuhan, Hubei, China \\ ${ }^{b}$ Huazhong University of Science and Technology, MoE Key Laboratory for Biomedical \\ Photonics, Wuhan, Hubei, China
}

\begin{abstract}
Three-dimensional visualization of the innervation in skeletal muscles is helpful for understanding the morphological structure and function. iDISCO, a whole-mount immunolabeling and clearing technique, provides a valuable tool for volume imaging of intramuscular nerve fibers but suffers from the nonspecific staining caused by the anti-mouse secondary antibody when using the murine primary antibody. We developed a modified iDISCO method by introducing pretreatment of ScaleCUBIC-1 reagent, termed m-iDISCO. The m-iDISCO method could eliminate the nonspecific staining and achieve uniform and complete labeling of nerve fibers in various muscles with mouse anti-neurofilament primary antibody. Combining the m-iDISCO method with light-sheet microscopy enabled us to visualize the innervation of adult mouse tibialis anterior and trace the nerve fibers from extramuscular branches to intramuscular terminal branches. This method represents an effective alternative for studying the innervation of intact skeletal muscles in health and disease. (c) The Authors. Published by SPIE under a Creative Commons Attribution 4.0 Unported License. Distribution or reproduction of this work in whole or in part requires full attribution of the original publication, including its DOI. [DOI: 10.1117/1.NPh.7.1.015003]
\end{abstract}

Keywords: skeletal muscle; whole-mount immunostaining; intramuscular innervation.

Paper 19078R received Jul. 30, 2019; accepted for publication Dec. 24, 2019; published online Jan. 22, 2020.

\section{Introduction}

Three-dimensional (3-D) visualization of intramuscular innervation can be particularly valuable for understanding morphological structures and functions of the skeletal muscles. ${ }^{1}$ Immunostaining on sectioned tissue is a powerful approach for labeling molecules of interest and revealing cellular distribution in many biological research studies, but it often requires extensive labor and time for 3-D reconstruction with potential artifacts. To circumvent these issues, whole-mount immunostaining methods have been developed to label intact large tissues, but it is still quite challenging to achieve uniform and complete labeling due to the difficulty of antibody penetration. ${ }^{2-6}$

In recent years, tissue optical clearing technique has emerged to render tissue transparent for deep-tissue imaging. ${ }^{7-11}$ Some of the optical clearing methods can increase not only optical transparency but also macromolecule-permeability of tissues, which can facilitate antibody penetration to a certain extent. Various tissue optical clearing methods, such as 3DISCO, CUBIC, SWITCH, and CLARITY, have been combined with whole-mount immunostaining for labeling and imaging of large-volume tissues. ${ }^{12-16}$ iDISCO, a simple method with no need of customized setup, enables facile detection of immunolabeled structures throughout not only intact embryos but also dense adult tissues. ${ }^{17}$

The mouse anti-neurofilament (anti-NF) antibody produced by Developmental Studies Hybridoma Bank (DSHB, 2H3) is a widely used primary antibody for immunolabeling of intramuscular nerve fibers. ${ }^{18,19}$ And this antibody is also much more cost-effective than other anti-NF antibodies. But the anti-mouse secondary antibody used after this murine primary antibody can cause nonspecific staining on the sarcolemma and the fascia surrounding the muscle and can

*Address all correspondence to Tingting Yu, E-mail: yutingting@hust.edu.cn 
further hinder entrance of remaining secondary antibody into the muscle. The iDISCO method provides an important tool for volume imaging of intramuscular nerve fibers. However, it is unable to decrease the nonspecific staining caused by the anti-mouse secondary antibody. ${ }^{17}$

In this study, we aim to modify the iDISCO method to achieve 3-D visualization of the innervation of skeletal muscles with murine anti-NF antibody. First, we introduced a pretreatment method to modify the iDISCO method, termed m-iDISCO, applied it to label various adult mouse skeletal muscles with the mouse anti-NF antibody (2H3, DSHB), then compared with the iDISCO method. Finally, using the modified method combining with light-sheet fluorescence microscope (LSFM), we conducted 3-D visualization of the innervation of adult mouse tibialis anterior.

\section{Materials and Methods}

\subsection{Animals}

In this study, adult (two- to three-month-old) Thyl-GFP-M mice (Jackson Laboratory) were used. All experimental procedures were performed in strict accordance with the Experimental Animal Management Ordinance of Hubei Province, China, and were approved by the Institutional Animal Ethics Committee of Huazhong University of Science and Technology.

\subsection{Sample Preparation}

Adult mice were deeply anesthetized with an intraperitoneal injection consisting of a mixture of $2 \%$ chloral hydrate and $10 \%$ urethane $(8 \mathrm{ml} / \mathrm{kg})$ and then transcardially perfused with $0.01 \mathrm{M}$ PBS (Sigma-Aldrich Co., St. Louis, Missouri) followed by $4 \%$ (w/v) paraformaldehyde (PFA, SigmaAldrich Co., St. Louis, Missouri) in 0.01 M PBS. The skeletal muscles were excised and postfixed overnight at $4^{\circ} \mathrm{C}$ in PFA, then the samples were washed with $0.01 \mathrm{M}$ PBS at least three times.

For muscle slices, the intact muscles were embedded in $4 \%(\mathrm{w} / \mathrm{v})$ agarose (Sigma-Aldrich Co., St. Louis, Missouri) and were cut with a vibratome (VT 1000s, Leica, Germany).

\subsection{Antibodies}

The antibodies used in this study included primary antibody: mouse anti-NF antibody (2H3, DSHB; dilution 1:100); secondary antibody: goat anti-mouse IgG Alexa Fluor 594 (115-585146, Jackson ImmunoResearch Laboratories, Inc.; dilution 1:300).

\section{$2.4 m$-iDISCO Protocol}

We developed the m-iDISCO method by introducing the pretreatment of ScaleCUBIC-1 reagent to modify the iDISCO method. Overview of the m-iDISCO protocol is shown in Fig. 1.

First, ScaleCUBIC-1 reagent was prepared as a mixture of $25 \mathrm{wt}$ \% urea (Sinopharm

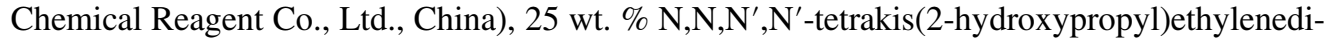
amine (Tokyo Chemical Industry Co., Ltd., Japan), and 15 wt. \% Triton X-100 (Sinopharm Chemical Reagent Co., Ltd., China) according to the original literature of CUBIC. ${ }^{12}$ The fixed muscle samples were immersed in ScaleCUBIC-1 reagent at $37^{\circ} \mathrm{C}$ with gentle shaking. The incubation time of ScaleCUBIC-1 reagent depended on the size of muscles (Table 1). The pretreated muscles were washed thrice within $3 \mathrm{~h}$ with 0.01 M PBS at room temperature with gentle shaking. Then, the muscles pretreated with ScaleCUBIC-1 reagent were performed methanol pretreatment, immunostaining, and tissue clearing by referring to the iDISCO protocol $;{ }^{17}$ the details are described below.

\subsubsection{Methanol pretreatment}

First, samples were dehydrated in methanol (Sinopharm Chemical Reagent Co., Ltd, China) solutions at ascending concentration gradient (50 vol. \%, 80 vol. \%, 100\%, and 100\% in 
Li et al.: Three-dimensional visualization of intramuscular innervation in intact adult skeletal muscle...

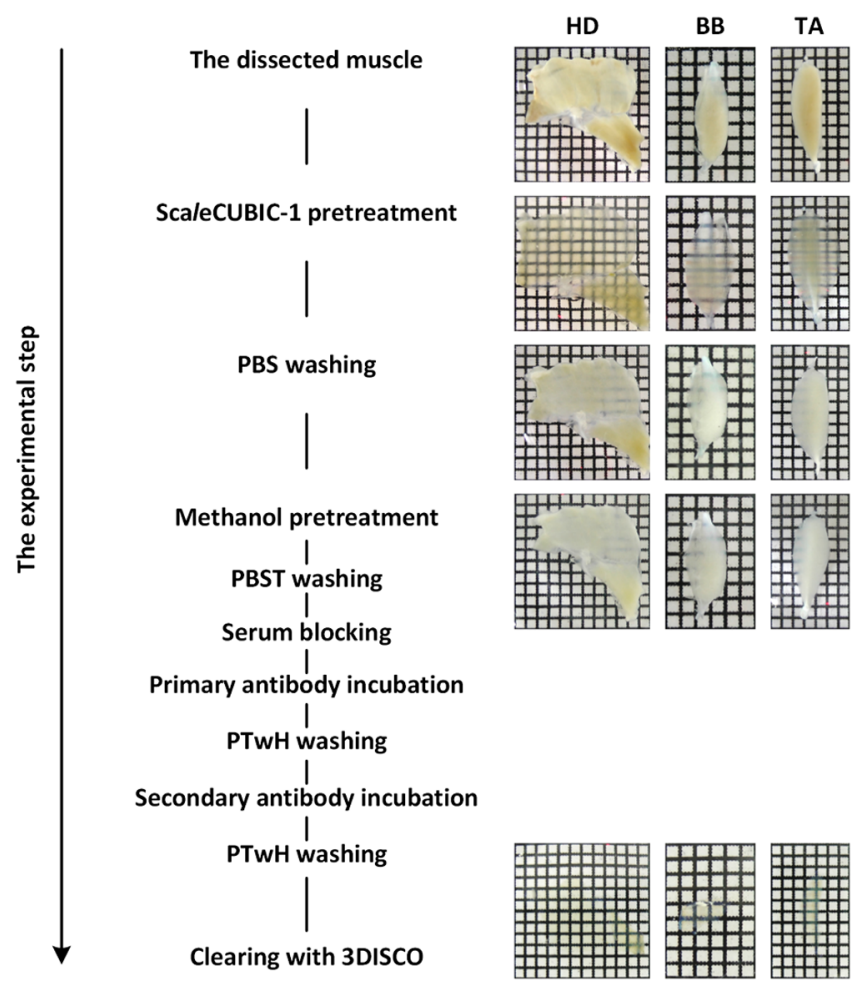

Fig. 1 Overview of the m-iDISCO protocol and bright-field images of adult mouse skeletal muscles during the experimental steps. HD, half diaphragm; BB, biceps brachii; TA, tibialis anterior.

Table 1 The time of main processing steps for different skeletal muscles.

\begin{tabular}{|c|c|c|c|c|}
\hline Muscle & $\begin{array}{c}\text { ScaleCUBIC-1 } \\
\text { pretreatment }\end{array}$ & $\begin{array}{l}\text { Serum } \\
\text { blocking }\end{array}$ & $\begin{array}{l}\text { Antibody } \\
\text { incubation }\end{array}$ & $\begin{array}{l}\text { Processing } \\
\text { time }^{\mathrm{a}}\end{array}$ \\
\hline Diaphragm & 1 day & 1 day & 3 days & 12 days \\
\hline Biceps brachii & 5 days & 1 day & 7 days & 24 days \\
\hline Tibialis anterior & 7 days & 1 day & 7 days & 26 days \\
\hline
\end{tabular}

aprocessing time: from sample collection to imaging.

$0.01 \mathrm{M}$ PBS) for $1 \mathrm{~h}$ /step. Samples were then bleached with $5 \% \mathrm{H}_{2} \mathrm{O}_{2}$ in $20 \%$ dimethylsulfoxide/ methanol (dimethylsulfoxide, DMSO, Sigma-Aldrich Co., St. Louis, Missouri; $30 \% \mathrm{H}_{2} \mathrm{O}_{2}$ : DMSO:methanol = 1:1:4 in volume) at $4^{\circ} \mathrm{C}$ overnight. After bleaching, samples were rehydrated in methanol solutions at descending concentration gradient (100\% and 100\%, 80 vol. \%, 50 vol. \% in $0.01 \mathrm{M}$ PBS) for $1 \mathrm{~h} / \mathrm{step}$. At last, samples were washed in 0.01M PBS twice within $2 \mathrm{~h}$. Except bleaching, other steps were conducted at room temperature.

\subsubsection{Immunolabeling protocol}

First, 0.2\% Triton X-100 (Sigma-Aldrich Co., St. Louis, Missouri) in 0.01 M PBS (PBST) and $0.2 \%$ Tween-20 (Sigma-Aldrich Co., St. Louis, Missouri) in $0.01 \mathrm{M}$ PBS with $10 \mathrm{mg} / \mathrm{ml}$ heparin $(\mathrm{PTwH})$ were prepared. After methanol pretreatment, samples were incubated in PBST with $0.3 \mathrm{M}$ glycine and $20 \% \mathrm{DMSO}$ at $37^{\circ} \mathrm{C}$ overnight, then blocked in PTwH with $6 \%$ goat serum and $10 \% \mathrm{DMSO}$ at $37^{\circ} \mathrm{C}$ for indicated time (Table 1). After blocking, samples were incubated in 
primary antibody diluted in PTwH with 3\% goat serum and 5\% DMSO for indicated time (Table 1). Then, samples were washed in PTwH for 1 day and incubated in secondary antibody diluted in PTwH with 3\% goat serum and 5\% DMSO for indicated time (Table 1). Samples were finally washed in PTwH for at least 1 day prior to clearing and imaging. Here, for the m-iDISCO and iDISCO methods, the antibody incubation and washing steps were conducted at room temperature.

\subsubsection{Tissue clearing}

Immunolabeled tissues were dehydrated in tetrahydrofuran (Sinopharm Chemical Reagent Co., Ltd., China) solutions at ascending concentration gradient (50 vol. $\%, 70$ vol. $\%, 80$ vol. $\%$, $100 \%$, and $100 \%$ in $\mathrm{dH}_{2} \mathrm{O}$ ). Finally, samples were incubated in dibenzyl ether (Shanghai Aladdin Bio-Chem Technology Co., Ltd., China) until cleared completely. The above experiments were carried out at room temperature. The diaphragms were dehydrated for $10 \mathrm{~min} / \mathrm{step}$ while the biceps brachii and the tibialis anterior were dehydrated for $1 \mathrm{~h} / \mathrm{step}$.

\subsection{Immunostaining of Muscle Slices}

To explore the nonspecific staining caused anti-mouse secondary antibody, we stained 300- $\mu \mathrm{m}$ thick muscle slices of the tibialis anterior only with the goat-anti-mouse antibody (115-585-146, Jackson ImmunoResearch).

First, muscle slices were blocked with $6 \%$ goat serum in $0.2 \%$ PBST for $1 \mathrm{~h}$. Then, the blocked muscle slices were directly incubated with the goat-anti-mouse secondary antibody diluted in $0.2 \%$ PBST with $3 \%$ goat serum (dilution 1:500). Finally, muscle slices were washed with $0.2 \%$ PBST six times during $30 \mathrm{~min}$.

\subsection{Imaging}

The diaphragms and muscle slices were imaged with an inverted confocal fluorescence microscope (LSM710, Zeiss, Germany) equipped with a $5 \times / 0.25$ dry objective (W.D. $12.5 \mathrm{~mm}$ ), a $10 \times / 0.5$ dry objective (W.D. $2.0 \mathrm{~mm}$ ), and a $20 \times / 0.8$ dry objective (W.D. $0.55 \mathrm{~mm}$ ).

The biceps brachii and the tibialis anterior were imaged with an LSFM (Ultramicroscope, LaVisionBioTec, Germany) equipped with a $2 \times / 0.5$ objective and a macro zoom body (magnification steps from $0.63 \times$ to $6.3 \times$ ).

When acquiring fluorescence images of samples stained with the iDISCO and m-iDISCO methods, respectively, we used the same acquisition settings.

\subsection{Data Processing}

The obtained images were analyzed with MATLAB, ImageJ, and Imaris software. MATLAB was used to calculate the signal and background intensity. ImageJ software was also used to perform maximum intensity projections (MIPs), image cropping and stitching, and analyze the grayscale values of pixels under marked line. Imaris software was used for 3-D and XZ-view reconstructions of z-stack images and tracing of nerve fibers within the muscles.

For the quantification of the fluorescence intensity in Fig. 2(d), we first obtained the MIPs of high-magnification image stacks of muscle sections by ImageJ software. Then, the MIPs were separated into the signal and background areas by a threshold function in MATLAB software. Finally, the mean intensities of all pixels in the two areas were calculated and defined as the signal and background intensity.

For the segmentation of nerve fibers in Fig. 5, we first converted images into an 8-bit format by ImageJ software to facilitate image processing. Then, we opened 8-bit stack images with Imaris software and applied the "filament" tool for nerve segmentation. We added the "filament" and drew each nerve filament manually from starting point to end point in the autodepth mode. In addition, we added several "filaments" and selected different colors to differentiate nerve branches. Finally, we use the "snapshot" and "animation" tools to generated 3-D pictures and movies. 


\section{Results}

\subsection{Elimination of Nonspecific Staining by ScaleCUBIC-1 Pretreatment}

As mentioned above, the mouse anti-NF primary antibody produced by DSHB (2H3) provides a cost-effective option for immunostaining of whole-mount skeletal muscles. However, the followed anti-mouse secondary antibody can abundantly bind to the dense sarcolemma and the fascia, especially for the ones with increased thickness in adult mouse skeletal muscles [Fig. 2(a)], which will substantially hinder penetration of antibodies and reduce the effective antibody binding. Although the iDISCO protocol can achieve whole-mount immunolabeling of various large tissues, ${ }^{17,20}$ it is unable to decrease the nonspecific binding when using this mouse anti-NF antibody. ScaleCUBIC-1 reagent, originated from CUBIC protocol, shows high delipidation efficiency in mouse brain and kidney (close to $50 \%)^{12,21}$ and has been widely used for rapid lipid removal prior to clearing or embedding. ${ }^{22,23}$ Here, we investigated the influence of ScaleCUBIC-1 processing of skeletal muscles on the nonspecific binding mentioned above [Fig. 2(b)]. We quantitatively compared the signal and background intensity without and with ScaleCUBIC-1 pretreatment [Figs. 2(c) and 2(d)]. The results showed that the pretreatment of ScaleCUBIC-1 reagent could weaken the binding between the anti-mouse secondary antibody with the sarcolemma and the fascia. Hence, we introduced the ScaleCUBIC-1 pretreatment to modify the iDISCO protocol to label adult skeletal muscles and developed the m-iDISCO method for 3-D visualization of the innervation in intact skeletal muscles.

\subsection{Whole-Mount Immunolabeling of Nerve Fibers in Adult Mouse Diaphragm}

To validate the effectiveness of the m-iDISCO protocol, we first applied it to label the nerve fibers in adult mouse diaphragm, a relatively thin skeletal muscle, and compared with the iDISCO method.

(a)
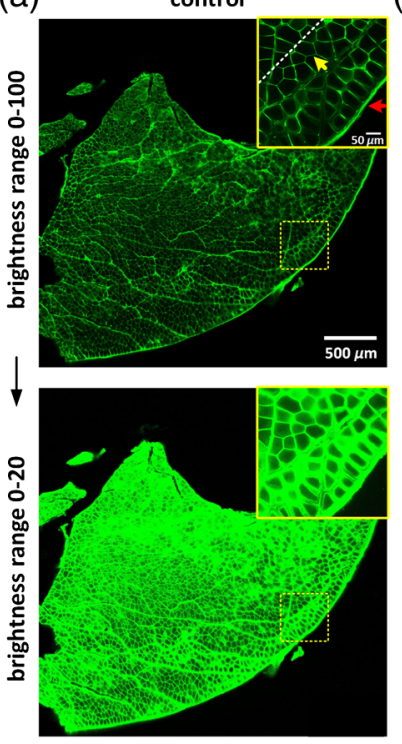

(b)
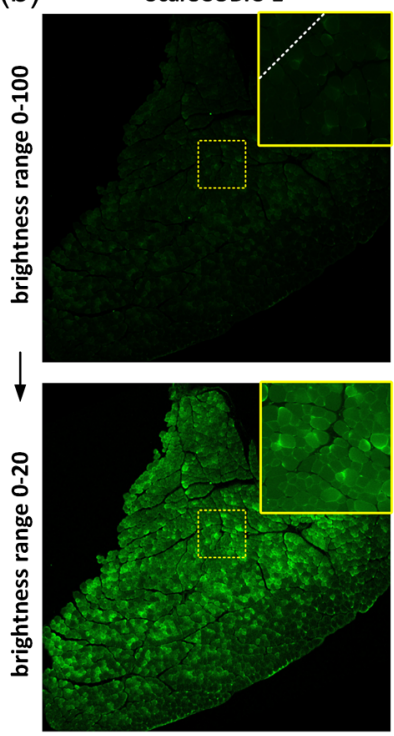

(c)

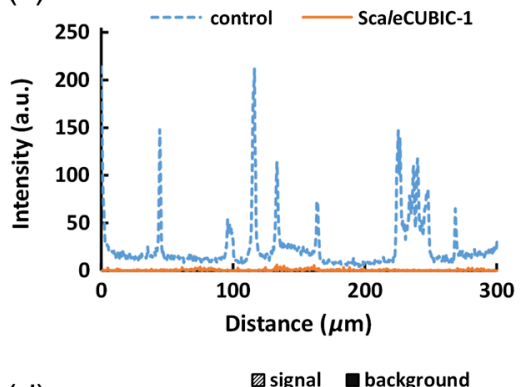

(d)

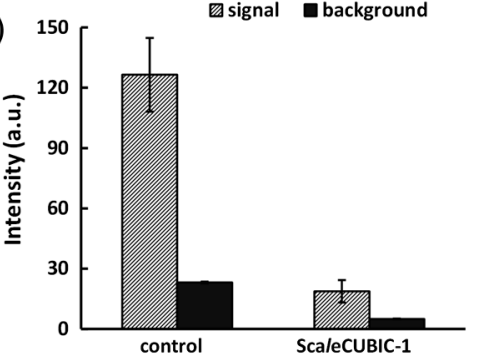

Fig. 2 The nonspecific staining of the goat-anti-mouse secondary antibody on 300- $\mu \mathrm{m}$-thick slices of adult mouse tibialis anterior. (a), (b) The 8-bit fluorescence images of muscle slices incubated with the secondary antibody. The muscle slices are from (a) fixed tibialis anterior and (b) one treated with ScaleCUBIC-1 reagent for 7 days, respectively. The red and yellow arrows show the nonspecific binding in the fascia and sarcolemma, respectively. The upper and bottom rows in (a) and (b) are from the same data, respectively. The brightness ranges were adjusted to 0 to 20 (bottom row) to clearly show the outlines of muscle slices. (c) Normalized intensity profiles of the marked regions in (a) and (b) with white dashed lines. (d) Quantification of the fluorescence intensity in the signal and background areas of high-magnification images in the inner parts of muscle slices ( $n=3$ for each condition). 
(a)

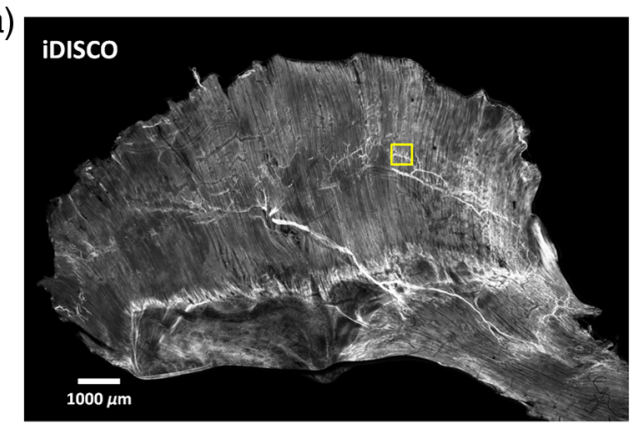

(c)

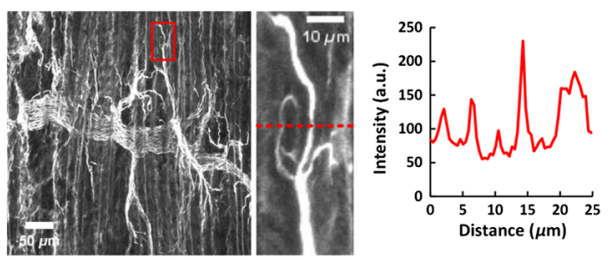

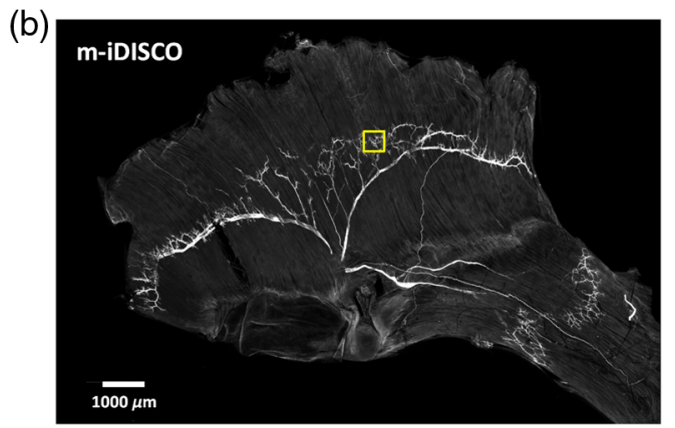

(d)

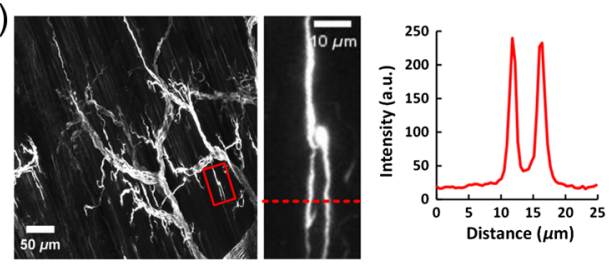

Fig. 3 Whole-mount immunostaining with the mouse anti-NF primary antibody on nerve fibers in mouse diaphragm. (a), (b) The MIPs of images of the immunolabeled adult diaphragms by (a) the iDISCO and (b) m-iDISCO methods, respectively. (c), (d) The high-magnification images of the regions in (a) and (b) with yellow boxes, respectively. The locations of nerve endings were indicated with the red boxes. The plots are the gray values of pixels along the red dashed lines in the enlarged images.

By the iDISCO method, the distribution of nerve fibers in the diaphragm was not clear due to the high level of background signal [Fig. 3(a)]. Although we tried to prolong the time of serum blocking, it did not lower the background staining on the surface of the diaphragm (Fig. S1). In contrast, the m-iDISCO method performed well on the diaphragm and showed complete staining with decreased background staining and clearly distinguishable trunks and branches of nerve fibers [Fig. 3(b)]. We observed the nerve branches and the nerve endings by the high-magnification images and obtained the intensity plots at the nerve endings [Figs. 3(c) and 3(d)]. The relative signal intensity in areas of the nerve endings revealed that the muscle stained with $\mathrm{m}$ iDISCO had lower background intensity and higher signal background ratio than the one stained with iDISCO. Hence, short-term ScaleCUBIC-1 pretreatment (1 day) in the m-iDISCO method lowered the background staining but did not reduce the labeling of nerves.

Notably, the samples shown in Fig. 2 were only stained with the secondary antibody to investigate the nonspecific binding effect, whereas the samples shown in Fig. 3 were stained with the primary antibody followed by the secondary antibody to label the nerves. The sarcolemma and the fascia labeled were regarded as the signal in Fig. 2, whereas they were regarded as the background in Fig. 3. Both Figs. 2 and 3 demonstrated that the labeling on the sarcolemma and the fascia could be reduced after ScaleCUBIC-1 pretreatment.

\subsection{Whole-Mount Immunolabeling of Nerve Fibers in Adult Mouse Biceps Brachii and Tibialis Anterior}

Furthermore, we compared the iDISCO method with the m-iDISCO method for labeling adult thick skeletal muscles. Here, we chose two typical muscles, including the biceps brachii from forelimb and the tibialis anterior from hind limb. After labeling, the muscles were imaged with LSFM and reconstructed with commercial software.

The 3-D reconstruction of the muscles stained with the iDISCO method showed that there were some nerve fibers detected on the bone surface of the muscles, but no detection inside of the muscles in the 3-D volumes [Figs. 4(a) and 4(b), Fig. S2(a)]. While for m-iDISCO-stained muscles, the distributions of nerve fibers in the inner part of the muscles were clearly presented [Figs. 4(a) and 4(b), Video 1]. We reconstructed XZ-view of the image stacks, obtained the $150-\mu \mathrm{m}$-thick MIPs of images in the middle of the muscles, and quantitatively measured the background intensity at the surface and the inside of the muscles [Figs. 4(c)-4(f); Fig. S2(b)]. 
(a)

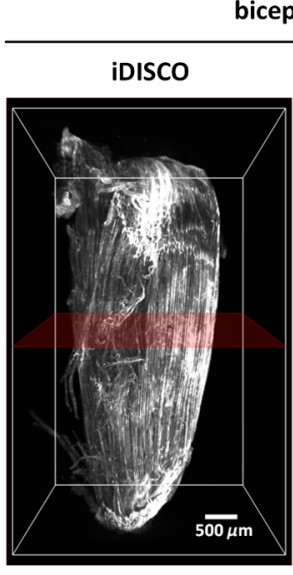

(c)

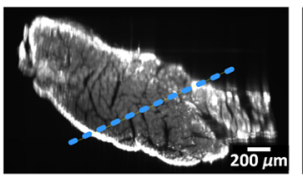

biceps brachii
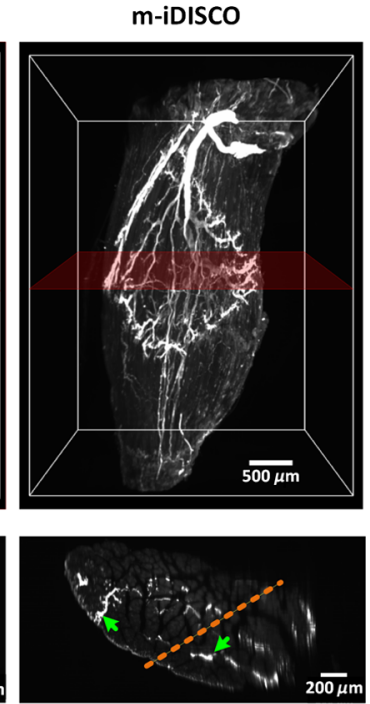

(b)

tibialis anterior

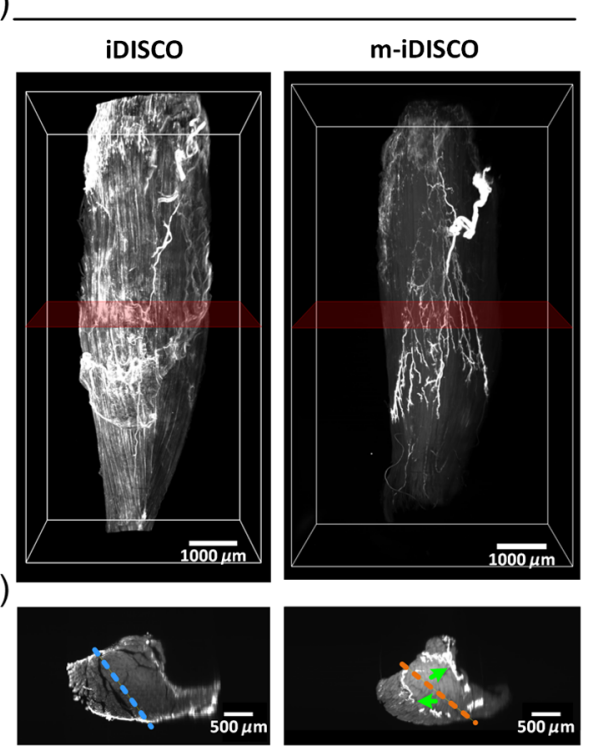

(e)

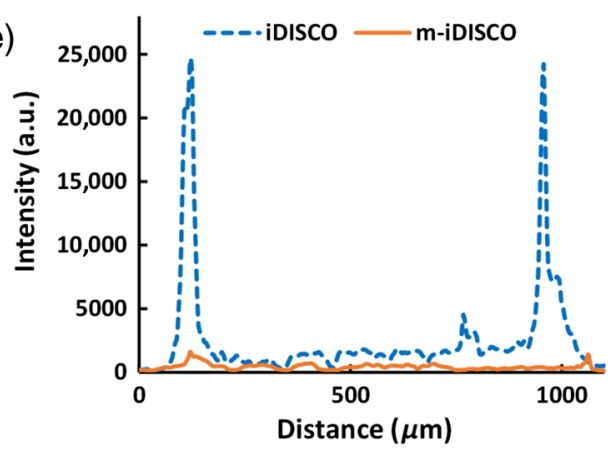

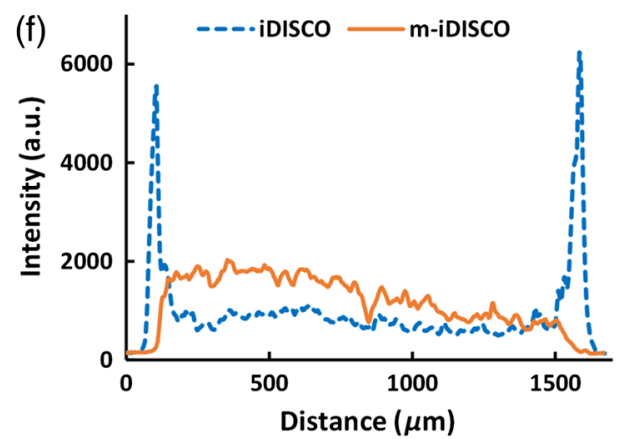

Fig. 4 Whole-mount immunostaining with the mouse anti-NF primary antibody on nerve fibers in adult mouse biceps brachii and tibialis anterior. (a), (b) 3-D reconstruction of the biceps brachii and the tibialis anterior, immunostained with the iDISCO and m-iDISCO methods, respectively (Video 1). (c), (d) The 150- $\mu$ m-thick MIPs of the images in $X Z$ direction. The rough locations are marked in (a) and (b) with translucent red frames, and the green arrows indicate the labeled nerve fibers. (e), (f) Normalized intensity profiles of the background signal at the surface and the inside of the muscles. The locations are marked in (c) and (d) with the dashed lines. (Video 1, MPEG, 11.6 MB [URL: https://doi.org/10.1117/1.NPh.7.1.015003.1]).

The results showed that, for iDISCO-stained muscles, the background intensity was higher on the surface than the inside of the muscles and the nerve fibers could not be distinguishable. In contrast, when stained with m-iDISCO, the background intensity was uniform for the entire muscles and the nerve fibers were labeled uniformly as indicated with the green arrows in Figs. 4(c) and 4(d).

The nonspecific binding between the anti-mouse secondary antibody and the muscles caused the background signal. As shown in Figs. 4(c)-4(f), the secondary antibody richly binded the fascia and the sarcolemma outside of the muscle, which made it difficult to penetrate into the inside when use the iDISCO method. While with the m-iDISCO method, the antibodies could effectively penetrate into the muscles to label the nerves inside owing to the pretreatment of ScaleCUBIC-1. Hence, ScaleCUBIC-1 pretreatment could not only weaken the nonspecific binding caused by the anti-mouse secondary antibody but also increase the penetration of the antibodies into the muscles for effective labeling. Notably, Figs. 4(e) and 4(f) showed that for the biceps brachii, m-iDISCO staining produced a relatively lower background signal inside the tissue than iDISCO, whereas for the tibialis anterior, $\mathrm{m}$-iDISCO staining produced a relatively higher background signal inside the tissue than iDISCO. We speculated that the biceps brachii got a fuller pretreatment than the tibialis anterior due to its smaller size, which induced a slightly 


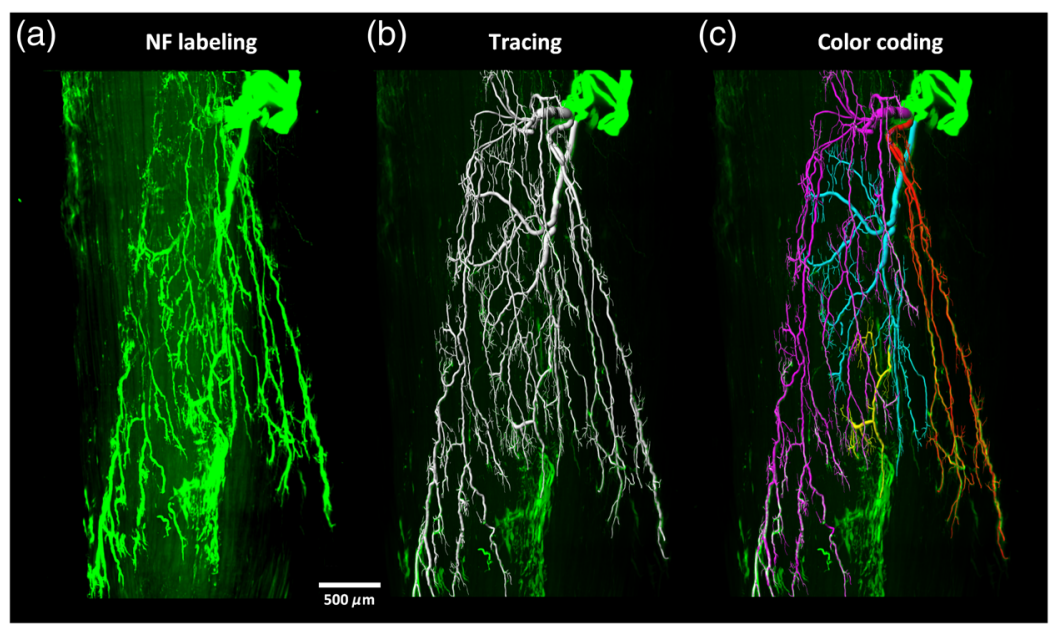

Fig. 5 3-D visualization of the motor nerve fibers in adult mouse tibialis anterior (Video 2). The tibialis anterior was immunolabeled and cleared with the m-iDISCO method, then imaged with LSFM. The images were segmented and the nerve branches were coded with different colors. (a) LSFM images, (b) segmentation, and (c) color coding of different branches. NF, neurofilament. (Video 2, MPEG, 11.2 MB [URL: https://doi.org/10.1117/1.NPh.7.1.015003.2]).

lower background signal with less binding between the sarcolemma inside the muscle with the anti-mouse secondary antibody.

\subsection{3-D Visualization of the Innervation in Adult Mouse Tibialis Anterior}

After m-iDISCO staining of adult tibialis anterior, we obtained the 3-D distribution of intramuscular innervation and traced the motor nerve fibers from extramuscular branches to intramuscular terminal branches (Fig. 5; Video 2). As known, the tibialis anterior is innervated by the deep peroneal nerve, which enters into the tibialis anterior from the posterior side. ${ }^{24,25}$ The $3-\mathrm{D}$ reconstruction and tracing of the nerve branches demonstrated that the motor nerve fibers in the tibialis anterior were distributed in an organized pattern of lamella, as shown with different colors in Fig. 5.

\section{Discussion}

Here, we developed a modified iDISCO method for 3-D visualization of intramuscular innervation in intact adult skeletal muscle, which we have called m-iDISCO. The m-iDISCO method achieved whole-mount anti-NF immunolabeling and clearing of intact adult mouse skeletal muscles, including diaphragm, biceps brachii, and tibialis anterior, and allowed 3-D analysis of intramuscular nerve fibers.

Notably, most studies focused on thin and small muscles of young mice in previous research due to the difficulty of labeling or imaging large muscles. ${ }^{18,26}$ Adult muscles are necessary objects when studying the muscle development and pathology. ${ }^{27}$ But the thickness of fascia surrounding the muscle increased and the tissues become denser with age, the antibody penetration into muscles of adult mice is more difficult than that of young mice. The modified protocol described in this work achieved whole-mount labeling of adult tibialis anterior (about 3-mm thick), which is thicker than muscles such as extensor digitorum longus ${ }^{28}$ and lumbricales. ${ }^{29}$ It is supposed that the pretreatment of ScaleCUBIC-1 reagent in the m-iDISCO method might eliminate hindrance of fascia and could facilitate antibody penetration.

In this study, we used a mouse anti-NF primary antibody to label the nerve fibers in skeletal muscles. The anti-mouse secondary antibody could bind to the sarcolemma and the fascia surrounding the mouse muscles. This rich nonspecific binding caused the strong background staining outside of the muscles, also hindered the entrance of the secondary antibody into muscles, leading to incomplete labeling. As described in above results, ScaleCUBIC-1 reagent could weaken this nonspecific labeling on skeletal muscles. A possible reason is the utilization of 
high-level detergent (15\%), Triton X-100, in ScaleCUBIC-1 reagent, which can maximize lipid removal and break down the cell membrane. ${ }^{7}$ However, for the same reason, ScaleCUBIC-1 pretreatment also risks lowering epitope concentrations and potentially weakens immunostaining. So the influence of ScaleCUBIC-1 pretreatment on the antigen-antibody reaction still needs to be verified before performing the m-iDISCO method with other antibodies.

In general, each experiment for screening appropriate antibodies requires extensive labor, time, and cost. This work demonstrated the labeling results with mouse anti-NF primary antibody ( $2 \mathrm{H} 3$, DSHB) by the m-iDISCO method, and these results can provide valuable references for the researchers. In addition, the mouse anti-NF primary (2H3, DSHB) used in this study is relatively cheaper ( $\sim \$ 0.4$ for a muscle, $\$ 40$ for $1 \mathrm{ml}$ ) than some other anti-NF primary antibodies (e.g., AB5539, Millipore, $\sim \$ 30$ for a muscle, $\$ 689$ for $50 \mu \mathrm{l}$ ). It provides a great and cost-effective option to study the innervation of various skeletal muscles in health and disease combined with the m-iDISCO method.

In summary, we have modified the iDISCO method by introducing the ScaleCUBIC-1 reagent for pretreatment. We obtained 3-D distribution of intramuscular nerve fibers in various skeletal muscles and conducted 3-D tracing of the innervation of adult mouse tibialis anterior. This method potentially provides an option for 3-D histological analysis of various skeletal muscles, facilitating to understand structural-functional relationship of skeletal muscle in physiological and pathological condition.

\section{Disclosures}

No conflicts of interest, financial or otherwise, are declared by the authors.

\section{Acknowledgments}

This work was based on an SPIE Proceedings paper previously published. ${ }^{30}$ This study was supported by the National Key Research and Development Program of China (Grant No. 2017YFA0700501), the National Natural Science Foundation of China (Grant Nos. 81701354, 61860206009,81870934 , and 31571002), the Science Fund for Creative Research Group of China (Grant No. 61721092), the Fundamental Research Funds for the Central Universities, HUST (Grant Nos. 2019KFYXMBZ069 and 2019KFYXJJS052), and the Open Research Fund of State Key Laboratory of Bioelectronics, Southeast University. We also thank the Optical Bioimaging Core Facility of WNLO-HUST for support with data acquisition.

\section{References}

1. A. Di Bona et al., "Towards a clearer view of sympathetic innervation of cardiac and skeletal muscles," Prog. Biophys. Mol. Biol. (2019).

2. R. V. Sillitoe and R. Hawkes, "Whole-mount immunohistochemistry: a high-throughput screen for patterning defects in the mouse cerebellum," J. Histochem. Cytochem. 50(2), 235-244 (2002).

3. T. Yokomizo et al., "Whole-mount three-dimensional imaging of internally localized immunostained cells within mouse embryos," Nat. Protoc. 7(3), 421-431 (2012).

4. J. A. Gleave et al., "A method for 3D immunostaining and optical imaging of the mouse brain demonstrated in neural progenitor cells," PLoS One 8(8), e72039 (2013).

5. M. Belle et al., "A simple method for 3D analysis of immunolabeled axonal tracts in a transparent nervous system," Cell Rep. 9(4), 1191-1201 (2014).

6. M. Belle et al., "Tridimensional visualization and analysis of early human development," Cell 169(1), 161-173.e12 (2017).

7. D. S. Richardson and J. W. Lichtman, "Clarifying tissue clearing," Cell 162(2), 246-257 (2015).

8. E. A. Susaki and H. R. Ueda, "Whole-body and whole-organ clearing and imaging techniques with single-cell resolution: toward organism-level systems biology in mammals," Cell Chem. Biol. 23(1), 137-157 (2016). 
9. T. Yu et al., "Optical clearing for multiscale biological tissues," J. Biophotonics 11(2), e201700187 (2018).

10. Y. Qi et al., "FDISCO: advanced solvent-based clearing method for imaging whole organs," Sci. Adv. 5(1), eaau8355 (2019).

11. I. Costantini et al., "In-vivo and ex-vivo optical clearing methods for biological tissues: review," Biomed. Opt. Express 10(10), 5251-5267 (2019).

12. E. A. Susaki et al., "Whole-brain imaging with single-cell resolution using chemical cocktails and computational analysis," Cell 157(3), 726-739 (2014).

13. A. Erturk et al., "Three-dimensional imaging of solvent-cleared organs using 3DISCO," Nat. Protoc. 7(11), 1983-1995 (2012).

14. E. Murray et al., "Simple, scalable proteomic imaging for high-dimensional profiling of intact systems," Cell 163(6), 1500-1514 (2015).

15. K. Chung et al., "Structural and molecular interrogation of intact biological systems," Nature 497(7449), 332-337 (2013).

16. S. Y. Kim et al., "Stochastic electrotransport selectively enhances the transport of highly electromobile molecules," Proc. Natl. Acad. Sci. U. S. A. 112(46), E6274-E6283 (2015).

17. N. Renier et al., "iDISCO: a simple, rapid method to immunolabel large tissue samples for volume imaging," Cell 159(4), 896-910 (2014).

18. J. N. Sleigh et al., "Morphological analysis of neuromuscular junction development and degeneration in rodent lumbrical muscles," J. Neurosci. Methods 227, 159-165 (2014).

19. T. R. Cheever, E. A. Olson, and J. M. Ervasti, "Axonal regeneration and neuronal function are preserved in motor neurons lacking $\beta$-actin in vivo," PLoS One 6(3), e17768 (2011).

20. N. Renier et al., "Mapping of brain activity by automated volume analysis of immediate early genes," Cell 165(7), 1789-1802 (2016).

21. K. Tainaka et al., "Chemical landscape for tissue clearing based on hydrophilic reagents," Cell Rep. 24(8), 2196-2210.e9 (2018).

22. M. N. Economo et al., "A platform for brain-wide imaging and reconstruction of individual neurons," Elife 5, e10566 (2016).

23. P. Matryba et al., "Optimized perfusion-based CUBIC protocol for the efficient whole-body clearing and imaging of rat organs," J. Biophotonics 11(5), e201700248 (2018).

24. C. K. Magill et al., "Reinnervation of the tibialis anterior following sciatic nerve crush injury: a confocal microscopic study in transgenic mice," Exp. Neurol. 207(1), 64-74 (2007).

25. D. Z. Yu et al., "Intramuscular innervations of lower leg skeletal muscles: applications in their clinical use in functional muscular transfer," Surg. Radiol. Anat. 38(6), 675-685 (2016).

26. T. Straka et al., "Postnatal development and distribution of sympathetic innervation in mouse skeletal muscle," Int. J. Mol. Sci. 19(7), 1935 (2018).

27. X. Yin et al., "Spatial distribution of motor endplates and its adaptive change in skeletal muscle," Theranostics 9(3), 734-746 (2019).

28. M. P. I. Williams et al., "A novel optical tissue clearing protocol for mouse skeletal muscle to visualize endplates in their tissue context," Front. Cell Neurosci. 13, 49 (2019).

29. A. C. Z. Rodrigues et al., "The sympathetic nervous system regulates skeletal muscle motor innervation and acetylcholine receptor stability," Acta Physiol. 225(3), e13195 (2019).

30. Y. S. Li et al., "An applicable whole-mount immunolabeling method for volume imaging of skeletal muscle," Proc. SPIE 10865, 108651E (2019).

Yusha Li received her bachelor's degree in biomedical engineering from China Medical University in 2015 and her MS degree in biomedical engineering from Wuhan National Laboratory for Optoelectronics of HUST in 2018, and she is pursuing her PhD there. Her main research interests are in vitro tissue optical clearing and three-dimensional immunofluorescence staining.

Jianyi Xu received his bachelor's degree in biotechnology from Huazhong Agricultural University in 2015. He is currently pursuing his $\mathrm{PhD}$ in biomedical engineering at Wuhan National Laboratory for Optoelectronics, HUST. His research interests include in vitro tissue optical clearing methods and fluorescence imaging of mouse peripheral nervous system. 
Jingtan Zhu received his bachelor's degree in optical information science and technology from Harbin Institute of Technology in 2015. He is currently pursuing his $\mathrm{PhD}$ in optical engineering at Wuhan National Laboratory for Optoelectronics, HUST. His research interest include in vitro tissue optical clearing methods and fluorescence imaging of biological tissues.

Tingting Yu received her doctoral degree in biomedical photonics from HUST in 2015, China. She is currently being an associate researcher at Wuhan National Laboratory for Optoelectronics, HUST. Her research activity is focused on tissue optical clearing method and neuroimaging with various optical imaging systems, including light-sheet microscopy and single/two-photon microscopy.

Dan Zhu received her PhD in physical electronics from HUST in 2001. She is currently a full professor of Wuhan National Optoelectronics Laboratory and a fellow of SPIE. She has engaged in the study of tissue optical imaging, including new theories, new technologies, and methods. Recently, her research interest is focused on tissue optical clearing of skin, skull, neuronal tissues, and other organs. 